\title{
Vigilância em saúde de populações expostas a agrotóxicos: revisão de escopo
}

\author{
Health surveillance of populations exposed to pesticides: scope review \\ Vigilancia sanitaria de las poblaciones expuestas a productos agroquímicos: examen del alcance
}

Recebido: 25/01/2021 | Revisado: 26/01/2021 | Aceito: 29/01/2021 | Publicado: 07/02/2021

\author{
Jéssica Cristina Ruths \\ ORCID: https://orcid.org/0000-0002-7400-1191 \\ Universidade Federal do Paraná, Brasil \\ E-mail: ruthsjessica@gmail.com \\ Francielle Brustolin de Lima Simch \\ ORCID: https://orcid.org/0000-0002-4722-4891 \\ Universidade Federal do Paraná, Brasil \\ E-mail: fbdlima@gmail.com
}

\begin{abstract}
Resumo
O Brasil é um dos países que mais consome agrotóxicos no mundo e o número de estudos que identificam e acompanham ações de saúde voltadas para populações cronicamente expostas ainda é incipiente. Assim, objetivou-se identificar as ações de vigilância em saúde orientadas para populações expostas a agrotóxicos implantadas no Brasil. Foi realizada uma Revisão de Escopo nas bases de dados Web of Science (WOS), Scopus, Biblioteca Virtual de Saúde (BVS), Scielo, Cochrane e Pub Med. Foram empregados os descritores "Public Health Surveillance", "Agrochemicals" e "Surveillance of the Workers Health". Onze artigos fizeram parte desta revisão, foi possível identificar ações de vigilância em saúde para populações expostas a agrotóxicos em nove artigos, dois não identificaram nenhuma atividade no âmbito avaliado. Entre as principais ações identificadas estão a notificação de intoxicações por agrotóxicos, suporte para diagnóstico e tratamento de intoxicações, uso de sistemas de informação para gerenciamento de informações, capacitação técnica de profissionais da saúde, monitoramento de contaminações de água e alimentos e elaboração de Planos de Ação Estadual para Vigilância de Populações Expostas a Agrotóxicos. A literatura indica que efetivar práticas de vigilância em saúde para este público envolve processos intersetoriais e interdisciplinares que incorporem nas análises situacionais locais o processo produtivo do agronegócio.
\end{abstract}

Palavras-chave: Estratégias locais; Órgãos dos sistemas de saúde; Vigilância em saúde pública.

\begin{abstract}
Brazil is one of the countries that most consumes pesticides in the world and the number of studies that identify and monitor health actions aimed at chronically exposed populations is still incipient. Thus, the objective was to identify health surveillance actions oriented to populations exposed to agrochemicals implemented in Brazil. A Scope Review was performed in the Web of Science (WOS), Scopus, Brazilian Virtual Health Library (BVS) ), Scielo, Cochrane and Pub Med databases. The descriptors "Public Health Surveillance", "Agrochemicals" and "Surveillance of the Workers Health" were used. Eleven articles were part of this review, it was possible to identify health surveillance actions for populations exposed to agrochemicals in nine articles, two did not identify any action in the scope evaluated. Among the main actions identified are the notification of pesticide poisoning, support for diagnosis and treatment of poisoning, use of information systems for information management, technical training of health professionals, monitoring of water and food contamination, and preparation of State Action Plans for Surveillance of Populations Exposed to Agrochemicals. The literature indicates that implementing health surveillance practices for this public involves intersectorial and interdisciplinary processes that incorporate the productive process of agribusiness into local situational analyses.
\end{abstract}

Keywords: Local strategies; Health systems organs; Public health surveillance.

\section{Resumen}

El Brasil es uno de los países que más plaguicidas consume en el mundo y el número de estudios que identifican y supervisan las medidas de salud destinadas a las poblaciones expuestas crónicamente es todavía incipiente. Así pues, el objetivo era identificar las acciones de vigilancia sanitaria dirigidas a las poblaciones expuestas a los agroquímicos que se aplicaban en el Brasil. Se realizó una revisión del alcance en las bases de datos Web of Science (WOS), Scopus, Biblioteca Virtual de Salud (BVS), Scielo, Cochrane y Pub Med. Se utilizaron los descriptores "Vigilancia de la salud pública", "Agroquímicos" y "Vigilancia de la salud de los trabajadores". Once artículos formaron parte de este examen, fue posible identificar acciones de vigilancia sanitaria para las poblaciones expuestas a agroquímicos en nueve artículos, dos no identificaron ninguna acción en el ámbito evaluado. Entre las principales acciones identificadas se encuentran la notificación de intoxicaciones por plaguicidas, el apoyo al diagnóstico y tratamiento de las intoxicaciones, el uso de sistemas de información para la gestión de la información, la capacitación técnica de los profesionales de la salud, la 
vigilancia de la contaminación del agua y los alimentos, y la preparación de planes de acción estatales para la vigilancia de las poblaciones expuestas a agroquímicos. La literatura indica que para que las prácticas de vigilancia sanitaria sean eficaces para este público se requieren procesos intersectoriales e interdisciplinarios que incorporen el proceso de producción de los agronegocios en los análisis de situación locales.

Palabras clave: Estrategias locales; Órganos de los sistemas de salud; Vigilancia de la salud pública.

\section{Introdução}

O Brasil é destaque mundial na produção agropecuária, em 2018, o setor gerou cerca de $\mathrm{R} \$ 1,4$ trilhão, que representaram 25\% do Produto Interno Bruto (PIB) brasileiro (CEPEA, 2020), já as exportações dos principais produtos agroindustriais somaram 43.803 milhões de dólares (Kreter \& Souza Júnior, 2018). Em termos de produção, foram cultivados 103 milhões de toneladas de soja e 173 milhões de cabeças de bovinos (IBGE, 2019). O setor ocupa grande parcela de trabalhadores em diversos ramos de atividade. Em 2017, foi responsável por empregar 19 milhões de pessoas (EMBRAPA, 2018).

O atual modelo de produção agropecuária, pauta-se em um projeto de desenvolvimento orientado por ganhos de produtividade. Alguns setores da cadeia produtiva são intensivos em capital, demandam o uso de tecnologias, máquinas, equipamentos, pesquisas e insumos, além de estar estreitamente dependente do uso de agrotóxicos (Abreu \& Tavares, 2016; Barros \& Barros, 2005).

Segundo o Sistema de Agrotóxicos Fitossanitários (Agrofit) do Ministério da Agricultura, Pecuária e Abastecimento (Mapa), entre 2007 e 2014 houve um aumento de 149\% na comercialização de agrotóxicos, enquanto isso, no mesmo período, a área plantada aumentou apenas 22,31\%, (Ministério da Saúde, 2018). A extensa área plantada e significativa produção, proporcionou que em 2008 o Brasil fosse considerado o maior consumidor de agrotóxicos do mundo (Carneiro, Augusto, Rigotto, Friedrich, \& Búrigo, 2015). Já em 2017, encontrava-se na quinta posição, atrás da China, Estados Unidos da América, Argentina e Tailândia (WorldAtlas, 2017).

Apesar da aplicação dos agrotóxicos aumentar a produtividade agrícola, o uso intensivo pode gerar externalidades negativas já documentadas na literatura especializada. Estes produtos produzem efeitos que variam conforme a forma de exposição, o princípio ativo e a dose absorvida (Ministério da Saúde, 2016; Silva, 2012) e impactam na qualidade do solo, água, nos alimentos e na saúde humana, causando intoxicações agudas e crônicas (Carneiro et al., 2015).

Apesar dos problemas decorrentes desse cenário serem de âmbito global, ações de enfrentamento requerem estratégias locais (Santos, Ruiz, Riquinho, \& Mesquita, 2015). Diversas estruturas políticas e sociais são fundamentais, devido a importância de análises situacionais que identifiquem os determinantes sociais e econômicos relacionados ao uso e exposição a agrotóxicos, aos processos históricos de uso do solo e da água, das condições edafoclimáticas e dos saberes e fazeres regionais das populações (Neves, Mendonça, Bellini, \& Pôssas, 2020).

Tendo em vista a existência de diversos territórios nacionais fortemente voltados para a agropecuária, o significativo uso de agrotóxicos e a relevância da problemática dos efeitos crônicos da exposição humana e ambiental a estes produtos, considera-se que o número de estudos que identificam e acompanham populações cronicamente expostas ainda é incipiente (Carneiro et al., 2015). Dessa forma, o presente estudo, mediante revisão de escopo, objetivou identificar as ações de vigilância em saúde orientadas para populações expostas a agrotóxicos implantadas no Brasil.

Adotou-se o conceito ampliado de vigilância em saúde, compreendida como um processo "contínuo e sistemático de coleta, consolidação, análise de dados e disseminação de informações sobre eventos relacionados à saúde" (Ministério da Saúde, 2018), implementados a fim de planejar e implementar medidas de saúde pública, nestas inclusas a atuação, intervenção e regulação nos condicionantes e determinantes da saúde para prevenção, controle de riscos, agravos e doenças e proteção e promoção da saúde (Ministério da Saúde, 2018). 
Considerando o modelo agrícola brasileiro, baseado na crescente demanda por agrotóxicos, a magnitude das exposições e a necessidade de intervenção sobre os riscos decorrentes do comércio, transporte, armazenamento, preparo, aplicação, dispersão e contaminação por agrotóxicos, quais devem ser desenvolvidas pelo Estado em todas as esferas de gestão, se faz relevante conhecer mais as atividades desenvolvidas pela vigilância em saúde em relação a temática (Neto, Lacaz, \& Pignati, 2014), a fim de identificar as potencialidades e fragilidades desse cenário.

\section{Metodologia}

Pesquisa de abordagem qualitativa empregando a Revisão de Escopo (Scoping Review) como artifício metodológico, conforme o método proposto pelo Instituto Joanna Briggs (JBI) (The Joanna Briggs Institute, 2015). Estudos qualitativos são empregados quando os dados geralmente são descritivos, a interpretação do pesquisador sobre o fenômeno é valorizada e a análise das informações tende a ser indutiva (Pereira et al., 2018). Já a revisão de escopo é utilizada para distinguir lacunas de conhecimento, investigar áreas de pesquisa e estruturar conceitos sobre assuntos específicos (The Joanna Briggs Institute, 2015).

Este estudo teve como etapas: (a) elaboração da pergunta de pesquisa; (b) identificação dos estudos relevantes; (c) seleção dos estudos; (d) mapeamento dos dados e (e) agrupamento, resumo e relação dos resultados (The Joanna Briggs Institute, 2015). Para a concepção da questão norteadora da pesquisa se utilizou a estratégia Population (P), Concept (C) e Context (C), (PCC) (The Joanna Briggs Institute, 2015), estabeleceram-se: P - Expostos a agrotóxicos; C - Ações de Vigilância em saúde; C - Brasil, ficando definida a pergunta de pesquisa: quais são as ações de vigilância em saúde orientadas para populações expostas a agrotóxicos no Brasil?

A pesquisa bibliográfica foi realizada nas plataformas Web of Science (WOS), Scopus, Biblioteca Virtual de Saúde (BVS), Scielo, Cochrane e Pub Med, durante o mês de setembro em 2020. Foi executada sem restrição de tempo, os dados foram analisados e selecionados pelo pesquisador principal e confirmados pelo segundo pesquisador autor deste trabalho, dúvidas e controvérsias foram analisadas e reavaliadas por todos os autores.

A escolha dos descritores foi realizada a partir dos Descritores em Ciências da Saúde (DeCS), com base a questão norteadora do estudo, utilizaram-se "Public Health Surveillance", "Agrochemicals" e "Surveillance of the Workers Health", o booleano "AND" foi utilizado para o cruzamento de descritores. As buscas foram realizadas com os descritores em língua inglesa.

Foram inclusos trabalhos que responderam à questão norteadora desta pesquisa, que estavam disponíveis na íntegra nas bases de dados selecionadas, em acesso aberto, escritos em inglês, português ou espanhol, de abordagem qualitativa e quantitativa, tendo como campo de estudo qualquer região do Brasil. Excluíram-se trabalhos que não responderam à questão norteadora, que estavam em duplicidade nas bases de dados, livros, guideline, teses e dissertações e estudos que foram realizados em outros países.

Primeiramente se avaliou as palavras contidas nos títulos e descritores, procedeu-se então a avaliação dos resumos e seleção de artigos para leitura na íntegra. As referências dos artigos lidos na íntegra foram analisadas em busca de novos estudos para compor a análise deste trabalho. Os artigos selecionados para fazer parte desta revisão tiveram os dados extraídos utilizando como ferramenta de coleta de dados o instrumento de coleta de revisão integrativa adaptado de Marziale (Marziale, 2015).

\section{Resultados}

Foram encontrados 236 estudos após a leitura de títulos e resumos, 50 trabalhos foram selecionados para leitura na íntegra, a avaliação de suas referências resultou na inclusão de mais seis artigos para esta etapa (Figura 1). Entre os escolhidos, 16 foram excluídos por se encontrarem em duplicidade nas bases de dados, quatro por se tratarem de livros e manuais e 25 por 
não responder à questão norteadora desta pesquisa, abordando as externalidades do consumo de agrotóxicos sem tratar das ações de vigilância em saúde para a população expostas e/ou potencialmente exposta.

A amostra final contemplou 11 artigos (Figura 1). A metodologia de busca e seleção dos artigos está representada no fluxograma (figura 1), conforme recomendações do JBI, segundo checklist adaptado do Preferred Reporting Items for Systematic Reviews and Meta-Analyses (PRISMA) (The Joanna Briggs Institute, 2015).

Figura 1 - Fluxograma do processo de seleção dos artigos adaptado do PRISMA.

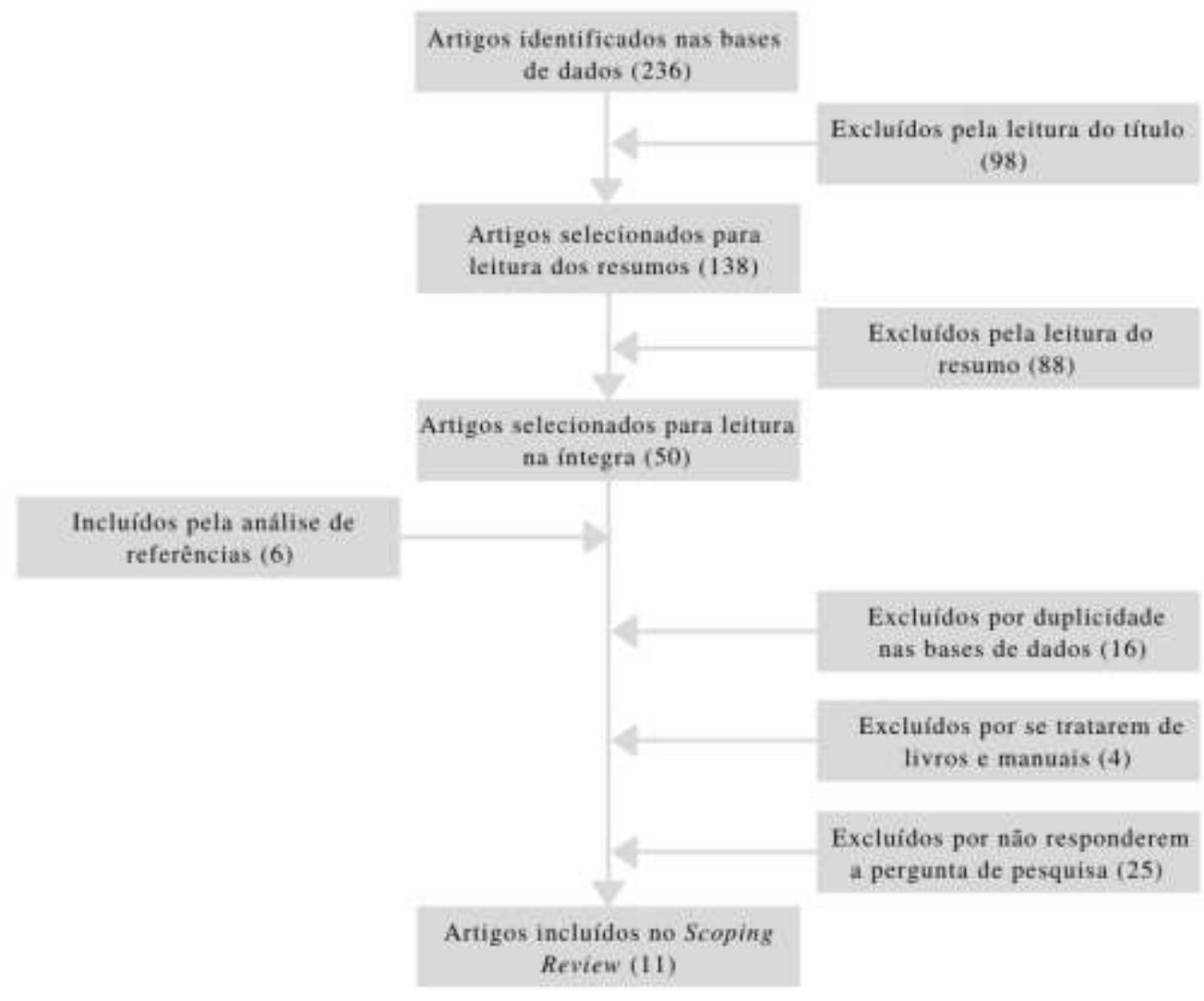

Fonte: Autores (2020).

Os 11 artigos inclusos nesta revisão foram publicados no período de 2007 (Faria, Fassa, \& Facchini, 2007) a 2020 (Freitas \& Garibotti, 2020; Neves et al., 2020; Silvério et al., 2020), todos se encontram em português. Quanto ao tipo de estudo foi identificada uma revisão de literatura (Abreu \& Tavares, 2016), um estudo de caso (Santos et al., 2015), três de campo (Neto et al., 2014; Silvério et al., 2020; Taveira \& Albuquerque, 2018), três ecológicos (Faria et al., 2007; Freitas \& Garibotti, 2020; Neves et al., 2020), dois relatos de experiência (Martins et al., 2016; Mota et al., 2016) e um ensaio (Rigotto et al., 2012). Todos tiveram como espaço empírico o território nacional, com objetos de estudo semelhantes, ações de vigilância em saúde voltadas para a população exposta a agrotóxicos, conforme itens caracterizados no Quadro 1. 
Quadro 1 - Caracterização dos estudos incluídos na revisão de acordo com autor principal, ano de publicação, local do estudo, tipo de estudo e resposta a questão norteadora da pesquisa.

\begin{tabular}{|c|c|c|c|}
\hline $\begin{array}{l}\text { Autor principal e ano } \\
\text { de publicação }\end{array}$ & $\begin{array}{l}\text { Local do } \\
\text { estudo }\end{array}$ & Tipo de estudo & Resposta à questão norteadora da pesquisa \\
\hline Faria et al., 2007. & $\begin{array}{l}\text { Rio Grande do } \\
\text { Sul, Brasil. }\end{array}$ & Ecológico & $\begin{array}{l}\text { - Ações de vigilância em saúde: uso sistemas oficiais para registro, } \\
\text { suporte de diagnóstico e tratamento de intoxicações por agrotóxicos, } \\
\text { sendo eles o Sistema Nacional de Informações Tóxico-Farmacológicas } \\
\text { (SINITOX), o Sistema Nacional de Agravos de notificação (SINAN) e } \\
\text { as Comunicações de Acidente de Trabalho (CAT), oferta de palestras de } \\
\text { atualização, elaboração de boletins periódicos e estimativas de base } \\
\text { populacionais sobre intoxicações por agrotóxicos; } \\
\text { - Fragilidades: subnotificação das intoxicações por agrotóxicos e } \\
\text { limitação de acesso a informações sobre o uso de agrotóxicos a partir } \\
\text { dos Receituários Agronômicos. }\end{array}$ \\
\hline Rigotto et al., 2012. & $\begin{array}{l}\text { Região do } \\
\text { Baixo } \\
\text { Jaguaribe, } \\
\text { Ceará, Brasil. }\end{array}$ & Ensaio & $\begin{array}{l}\text { - Não foram identificadas ações de vigilância em saúde relacionadas aos } \\
\text { agrotóxicos no sistema público avaliado; } \\
\text { - Fragilidades: reduzida capacidade instalada para o diagnóstico, } \\
\text { tratamento e notificação dos casos de intoxicação aguda e crônica por } \\
\text { agrotóxicos. }\end{array}$ \\
\hline Neto et al., 2014. & $\begin{array}{l}\text { Mato Grosso, } \\
\text { Brasil. }\end{array}$ & Campo & $\begin{array}{l}\text {-Ações de vigilância em saúde: notificação das intoxicações por } \\
\text { agrotóxicos por todos os municípios do estudo e, alguns municípios } \\
\text { desenvolvem o programa ambiental Vigi-água e Vigi-ar; } \\
\text { - Fragilidades: subnotificação de intoxicações por agrotóxicos, } \\
\text { dificuldades no controle do uso de Receituário Agronômico, número } \\
\text { reduzido de profissionais nas equipes, falta de capacitação técnica e } \\
\text { ausência de interesse político para efetivação de ações. }\end{array}$ \\
\hline Santos et al., 2015. & $\begin{array}{l}\text { Rio Grande do } \\
\text { Sul, Brasil. }\end{array}$ & Estudo de caso. & $\begin{array}{l}\text { - Ações de vigilância em saúde: notificação de intoxicações por } \\
\text { agrotóxicos; } \\
\text { - Fragilidades: subnotificação de intoxicações por agrotóxicos, carência } \\
\text { de indicadores ambientais e de intoxicações, falta de pessoal para atuar } \\
\text { na vigilância em saúde, ausência de capacitação técnica para } \\
\text { profissionais de saúde em dois municípios e desinteresse político para } \\
\text { efetivação de ações; } \\
\text { - Potencialidades: investimento em capacitação técnica dos profissionais } \\
\text { de saúde e parceria da Secretaria de Saúde com a Empresa de Assistência } \\
\text { Técnica e Extensão Rural (EMATER), em um dos municípios avaliados. }\end{array}$ \\
\hline Abreu \& Tavares, 2016. & Bahia, Brasil. & $\begin{array}{l}\text { Revisão } \\
\text { Literatura }\end{array}$ & $\begin{array}{l}\text { - Ações de vigilância em saúde: atuação do Sistema de Informação de } \\
\text { Vigilância da Qualidade da Água para Consumo Humano (Sisagua) e do } \\
\text { Programa de Análise de Resíduos de Agrotóxicos em Alimentos } \\
\text { (PARA) e uso dos sistemas de informação da CAT, SINITOX, SINAN, } \\
\text { Sistema de Informação de Mortalidade (SIM) e Sistema de Informações } \\
\text { Hospitalares (SIH) para notificação de intoxicações por agrotóxicos e } \\
\text { obtenção de dados; } \\
\text { - Fragilidades: subnotificação de intoxicações por agrotóxicos. }\end{array}$ \\
\hline Martins et al., 2016. & Bahia, Brasil. & $\begin{array}{l}\text { Relato } \\
\text { experiência }\end{array}$ & $\begin{array}{l}\text { - Ações de vigilância em saúde: elaboração do Plano de Ação Estadual } \\
\text { para Vigilância de Populações Expostas a Agrotóxicos, implementação } \\
\text { do curso para técnicos da vigilância em saúde dos municípios, dos } \\
\text { centros de referências em saúde do trabalhador e das Diretorias } \\
\text { Regionais de Saúde e a construção de fluxos de atenção a saúde e } \\
\text { vigilância em saúde de populações expostas a agrotóxicos; } \\
\text { - Potencialidades: educação em saúde para a população exposta, } \\
\text { capacitação técnica de profissionais de saúde e parcerias intersetoriais; } \\
\text { - Fragilidades: pouca governabilidade do setor saúde na regulação do } \\
\text { uso dos agrotóxicos. }\end{array}$ \\
\hline Mota et al., 2016. & Bahia, Brasil. & $\begin{array}{l}\text { Relato } \\
\text { experiência }\end{array}$ & $\begin{array}{l}\text { - Ações de vigilância em saúde: investigação da situação de intoxicação } \\
\text { por Benzoato de Emamectina, inspeção in loco, emissão de pareceres e } \\
\text { recomendações de ações para proteção da saúde dos trabalhadores e da }\end{array}$ \\
\hline
\end{tabular}




\begin{tabular}{|c|c|c|c|}
\hline & & & $\begin{array}{l}\text { população em geral, capacitação técnica para diagnóstico e manejo de } \\
\text { intoxicações, elaboração de materiais educativos para profissionais de } \\
\text { saúde, produtores rurais e população em geral; } \\
\text { - Potencialidades: elaboração de desenvolvimento de estratégias de } \\
\text { forma intersetorial. }\end{array}$ \\
\hline $\begin{array}{l}\text { Taveira \& Albuquerque, } \\
2018 .\end{array}$ & Paraná, Brasil & Campo & $\begin{array}{l}\text { - Ações de vigilância em saúde: notificação de intoxicações por } \\
\text { agrotóxicos; } \\
\text { - Fragilidades: desconhecimentos dos profissionais da vigilância em } \\
\text { saúde sobre o número de notificações de intoxicações por agrotóxicos, } \\
\text { subnotificação e baixa capacitação dos profissionais. }\end{array}$ \\
\hline Neves et al., 2020. & Goiás, Brasil. & Ecológico & $\begin{array}{l}\text { - Ações de vigilância em saúde: registro de intoxicações por agrotóxicos, } \\
\text { direcionamento de seu diagnóstico e tratamento, acompanhamento } \\
\text { telefônico de sua evolução e disponibilização de informações sobre as } \\
\text { intoxicações; } \\
\text { - Fragilidades da vigilância em saúde: falta de articulação entre os } \\
\text { pontos da rede de atenção à saúde, subnotificação de intoxicações por } \\
\text { agrotóxicos e déficit na completude das informações das fichas de } \\
\text { notificação. }\end{array}$ \\
\hline Silvério et al. 2020. & $\begin{array}{l}\text { Minas Gerais, } \\
\text { Brasil. }\end{array}$ & Campo & $\begin{array}{l}\text { - Não foram identificadas ações específicas de vigilância em saúde para } \\
\text { população exposta a agrotóxicos no cenário avaliado. }\end{array}$ \\
\hline $\begin{array}{l}\text { Freitas \& Garibotti, } \\
2020 .\end{array}$ & $\begin{array}{l}\text { Rio Grande do } \\
\text { Sul. }\end{array}$ & Ecológico & $\begin{array}{l}\text { - Ações de vigilância em saúde: criação do grupo técnico (GT } \\
\text { agrotóxicos), para elaborar e propor ações para o Programa Estadual de } \\
\text { Vigilância em saúde de Populações Expostas aos Agrotóxicos e } \\
\text { notificação de intoxicações por agrotóxicos; } \\
\text { - Fragilidades: déficit na completude das informações das fichas de } \\
\text { notificação; } \\
\text { - Potencialidades: aumento no número de registros das intoxicações por } \\
\text { agrotóxicos. }\end{array}$ \\
\hline
\end{tabular}

Fonte: Autores.

A partir das análises dos artigos que integraram esta revisão foi possível identificar ações de vigilância em saúde para populações expostas a agrotóxicos em nove artigos (Abreu \& Tavares, 2016; Faria et al., 2007; Freitas \& Garibotti, 2020; Martins et al., 2016; Mota et al., 2016; Neto et al., 2014; Neves et al., 2020; Santos et al., 2015; Taveira \& Albuquerque, 2018). apenas dois (Rigotto et al., 2012; Silvério et al. 2020) não identificaram nenhuma ação no âmbito avaliado. Entre as principais ações identificadas estão a notificação de intoxicações por agrotóxicos (Abreu \& Tavares, 2016; Faria et al., 2007; Freitas \& Garibotti, 2020; Neves et al., 2020; Neto et al., 2014; Santos et al., 2015; Taveira \& Albuquerque, 2018), o suporte para diagnóstico e tratamento de intoxicações (Faria et al., 2007; Neves et al., 2020), uso de sistemas de informação para gerenciamento de informações (Abreu \& Tavares, 2016; Freitas \& Garibotti, 2020; Neves et al., 2020), capacitação técnica de profissionais da saúde (Martins et al., 2016; Mota et al., 2016), monitoramento de contaminações de água e alimentos (Abreu \& Tavares, 2016; Neto et al., 2014) e elaboração de Planos de Ação Estadual para Vigilância de Populações Expostas a Agrotóxicos (Freitas \& Garibotti, 2020; Martins et al., 2016).

Enquanto potencialidades, verificou-se o investimento em capacitação técnica para profissionais da saúde (Martins et al., 2016; Santos et al., 2015), a educação em saúde para a população exposta (Martins et al., 2016), a instituição de parcerias intersetoriais (Martins et al., 2016; Mota et al., 2016; Santos et al., 2015) e o aumento do número das notificações de intoxicações por agrotóxicos (Freitas \& Garibotti, 2020).

Entre as principais fragilidades, apontam-se as subnotificações de intoxicações por agrotóxicos (Abreu \& Tavares, 2016; Faria et al., 2007; Neto et al., 2014; Neves et al., 2020; Santos et al., 2015; Taveira \& Albuquerque, 2018), déficit de completitude das fichas de notificação de intoxicação (Freitas \& Garibotti, 2020; Neves et al., 2020), o número reduzido de profissionais trabalhando na vigilância em saúde (Neto et al., 2014; Rigotto et al., 2012; Santos et al., 2015), falta de capacitação técnica (Neto 
et al., 2014; Santos et al., 2015; Taveira \& Albuquerque, 2018), limitações no acesso a dados de informação sobre o uso de agrotóxicos (Faria et al., 2007; Neto et al., 2014; Santos et al., 2015) e a falta de interesse político para efetivação das ações (Neto et al., 2014; Santos et al., 2015).

\section{Discussão}

Os artigos incluídos nesta revisão indicam que as ações de vigilância em saúde, específicas para populações expostas a agrotóxicos, quando existem efetivamente, priorizam a notificação, diagnóstico e tratamento de intoxicações, monitoramento da qualidade da água e de contaminação alimentar, o uso de sistemas de informações e as capacitações técnicas sobre intoxicações por agrotóxicos em detrimento das demais atividades. Ressalta-se que ações realizadas in loco, para identificação da exposição e recomendações de ações foram verificadas apenas no estudo de Mota et al. (2016).

Além das ações supracitadas, é importante que a as atividades da vigilância em saúde para população exposta a agrotóxicos englobem o reconhecimento de todos os processos através dos quais as populações se contaminam, incluindo a venda, transporte, armazenamento, preparo, uso, dispersão e descarte de embalagens, avaliem e orientem o emprego de equipamento de proteção individual, reconheçam a quantidade e os tipos de agrotóxicos utilizados na região, monitorem os efeitos à saúde e realizem atividades educativas e ações intersetoriais (Abreu \& Tavares, 2016; Ministério da Saúde, 2016; Neto et al., 2014).

Considerando os cenários avaliados nos artigos, pode-se dizer que na vigilância em saúde para populações expostas agrotóxicos, ainda há displicência na atuação sobre os determinantes ambientais e de trabalho na saúde, sobretudo em relação a identificação e redução de riscos. De acordo as Diretrizes Nacionais para a Vigilância em saúde de Populações Expostas a Agrotóxicos, quanto mais eficientes forem as estratégias em âmbito local, melhores serão as chances de que as ações de controle e uso adequado sejam incorporadas pela população (Ministério da Saúde, 2016). Riggoto et al. (2012) enfatiza que cada território é singular, portanto, caracterizar a existência do risco, a extensão e diversidade do uso dos agrotóxicos e as fragilidades das práticas de manejo já deveria ser rotina nas práticas de territorialização de saúde a fim de desencadear ações de vigilância a partir destes dados. Portanto, espera-se que a atuação local não se reduza a atenção as intoxicações, fato identificado em diversos estudos (Neto et al., 2014; Neves et al., 2020; Santos et al., 2015; Taveira \& Albuquerque, 2018).

Supõe-se que regiões com economia essencialmente agrícola, que façam uso de considerável quantidade de agrotóxicos, precisam desenvolver políticas públicas com foco específico nas populações expostas e seus determinantes (Neto et al., 2014). De acordo com Lacaz, Machado e Porto (2002) os serviços de saúde implementados não deveriam se limitar as ações ofertadas tradicionalmente na Atenção Primária em Saúde (APS), são necessárias políticas que rompam com o modelo hospitalocêntrico e desenvolvam ações intersetoriais, centradas na promoção da saúde, prevenção de agravos e na vigilância em saúde.

Neste sentido, desde 2002, o Ministérios da Saúde desenvolve a Vigilância em Saúde de Populações Expostas a Agrotóxicos (VSPEA), objetivando a implementação de ações integradas destinadas as populações expostas ou potencialmente expostas a agrotóxicos. Sua implantação foi incentivada pela publicação da Portaria do MS/GM nº 2.938 , de 22 de dezembro de 2012, que autorizou o repasse de recursos do Fundo Nacional de Saúde aos Fundos Estaduais de Saúde e do Distrito Federal para o fortalecimento da VSPEA. Após o recebimento dos recursos o Ministério da Saúde incentivou que todas as unidades de federação, elaborassem suas propostas de ações da VSPEA, de modo a refletir o compromisso com o desenvolvimento e acompanhamento de ações para a população exposta (Ministério da Saúde, 2018).

A VSPEA compreende um conjunto de ações integradas que devem envolver a proteção e promoção da qualidade de vida, prevenção de agravos e redução, controle ou eliminação da vulnerabilidade e dos riscos à saúde da população exposta (Ministério da Saúde, 2016; Resolução № 588, 2018). Esse processo compreende a coleta, processamento, análise e interpretação dos dados, com ênfase nas atividades econômicas do território e mapeamento dos riscos, incluindo as atividades produtivas 
relacionadas à exposição a agrotóxicos, análise da situação de saúde da população exposta, cadastramento de áreas de exposição, priorização de locais de risco, recomendações e implementação de ações apropriadas, avaliação a eficácia e efetividade das medidas adotadas e divulgação das informações pertinentes (Ministério da Saúde, 2016).

Os desafios para o avanço da VSPEA envolvem a integração de campos de saberes e competências de diversos setores do governo, bem como da articulação com os setores privados, não governamentais e a sociedade organizada com o propósito de instituir compromissos em favor da qualidade de vida das populações expostas e/ou potencialmente expostas a agrotóxicos (Ministério da Saúde, 2016).

Cabe ainda destacar que segundo a Lei Orgânica da Saúde nº. 8080/90 o Estado, nas três esferas de gestão, deve cumprir seu papel de proteger a saúde de toda a população, conciliando as atividades econômicas ao estado de saúde. Especificamente, o Ministério da Saúde e as Secretarias Estaduais e Municipais de Saúde devem se incumbir de garantir a integralidade da atenção à saúde através de toda a rede de saúde do Sistema Único de Saúde (Lei no 8.080,1990).

Entre as ações de saúde identificadas nesta revisão, uso de sistemas de informação para o registro de notificações de intoxicação por agrotóxicos e outros fins, foram as que mais se destacaram. Os trabalhos de Abreu e Tavares (2016) e Faria et al. (2007) relatam o uso do SINITOX para direcionamento dos diagnósticos e tratamentos de intoxicações por agrotóxicos, outros estudos identificaram o uso do sistema com a mesma finalidade (Garcia \& Alves Filho, 2005; Pires, Caldas, \& Recena, 2005).

Percebe-se relevância do sistema enquanto suporte toxicológico, principalmente para os profissionais que atendem casos agudos de intoxicação. Contudo, há distribuição desigual dos Centros de Informação Toxicológicas, responsáveis por esse sistema, no território brasileiro, a região nordeste por exemplo, possui apenas dois, enquanto a sudeste possui 16, além disso os centros se concentram nas capitais dos estados (Faria et al., 2007). Isso sugere que estados com maior número de centros podem ter melhores registro de dados (Oliveira, Silva, Ballani, \& Bellasalma, 2003), enquanto nos demais pode haver maior quantidade de subnotificação.

Outro sistema de registro de intoxicações com uso comumente identificado pelos estudos é o SINAN (Abreu \& Tavares, 2016; Faria et al., 2007; Freitas \& Garibotti, 2020; Neves et al., 2020; Taveira \& Albuquerque, 2018), sistema onde deveriam ser notificados todos os casos de intoxicação por agrotóxicos da rede pública. Apesar da melhoria no processo de notificação identificado por Freitas e Garibotti (2020), sabe-se a subnotificação ainda é expressiva no Brasil, em especial nos casos de intoxicação crônica. Na prática se registram prioritariamente casos agudos e graves (Faria et al., 2007), estima-se que apenas $20 \%$ das intoxicações são registradas, apesar de serem eventos de notificação compulsória (Taveira \& Albuquerque, 2018).

A subnotificação das intoxicações por agrotóxicos foi identificada como uma fragilidade da vigilância em saúde em cinco trabalhos desta revisão (Abreu \& Tavares, 2016; Faria et al., 2007; Neto et al., 2014; Neves et al., 2020; Santos et al., 2015; Taveira \& Albuquerque, 2018;). Demais estudos também têm demonstrado a magnitude da subnotificação (Albuquerque, Gurgel, Gurgel, Augusto, \& Siqueira, 2015; Jorge, Laurenti, \& Gotlieb, 2010). Os motivos relacionados a ela envolvem a baixa procura de atendimento por parte da população sintomática, falha no diagnóstico, não notificação de casos diagnosticados, falta de estruturação dos serviços de saúde e a ausência ou baixa implementação das ações de vigilância em saúde (Abreu \& Tavares, 2016; Taveira \& Albuquerque, 2018).

Soma-se a isso a problemática da completitude das fichas de notificação (Freitas \& Garibotti, 2020; Neves et al., 2020). Freitas e Garibotti (2020), encontraram variação de 66 a 100\% na completitude das informações com relação às variáveis analisadas nas fichas de notificação, corroborando Albuquerque et al. (2015), também identificaram incompletude de dados essenciais para o monitoramento e vigilância da população exposta a agrotóxicos, principalmente relacionados a ocupação e escolaridade dos intoxicados. Possivelmente o não preenchimento ou preenchimento errôneo pode estar relacionado ao despreparo da equipe que notifica os casos (Freitas \& Garibotti, 2020). Este déficit de informações dificulta o dimensionamento do problema para o país, além de ocultar os custos desses atendimentos para o Sistema Único de Saúde. 
Quanto as comunicações destes agravos na CAT, ação verificada nos trabalhos de Abreu e Tavares (2016) e Faria et al. (2007), tem-se a limitação da restrição do registro apenas para trabalhadores formais e a subnotificação no sistema. Em estudo realizado com agricultores familiares, no Rio Grande do Sul, as intoxicações por agrotóxicos eram equivalentes a 16\% dos acidentes de trabalho, contudo a CAT foi emitida em somente 4\% dos casos (Faria, Facchini, Fassa, \& Tomasi, 2004).

Salienta-se a importância de sistemas de informação que registram e armazenam dados, que possuem potencial para gerar de forma sistematizada, oportuna e constante informações para contribuir com formulação de políticas, tomada de decisões, planejamento, execução e avaliações de ações de vigilância em saúde (Ministério da Saúde, 2016).

Outra ação de vigilância em saúde detectada foi a capacitação técnica da equipe de saúde (Martins et al., 2016; Mota et al., 2016). Esta estratégia é considerada uma ferramenta de gestão com potencial para desenvolver a capacidade criativa de enfretamento de situações de saúde, além de reflexões e mudanças no cotidiano dos serviços, através de um processo que ocorre do trabalho para o trabalho (Ministério da Saúde, 2012). Além disso, os profissionais de saúde passam a assumir o compromisso ético com as populações que trabalham a partir do momento em propõem formulações e aplicações de políticas para incidir sobre os determinantes sociais em setores prioritários (Pignati, Oliveira, \& Silva, 2014).

A baixa capacitação técnica da equipe de saúde fragilizou as ações da vigilância em saúde (Neto et al., 2014; Santos et al., 2015; Taveira \& Albuquerque, 2018), enquanto as atividades de capacitação realizadas foram consideradas fortalecedoras (Martins et al., 2016; Santos et al., 2015). O investimento em educação continuada para profissionais de saúde sensibiliza em relação a magnitude dos problemas advindos da exposição, capacita os trabalhadores para avaliar as condições de condições de trabalho, além de ser capaz de desacomodar as práticas assistenciais em função de uma reflexão crítica para o compromisso com a saúde ambiental (Beserra, Alves, Pinheiro, \& Vieira, 2010; Hendges et al., 2019).

Quanto as ações de monitoramento da água e de alimentos (Abreu \& Tavares, 2016; Neto et al., 2014), o Programa de Análise de Resíduos de Agrotóxicos em Alimentos (PARA) analisou 1.628 amostras de alimentos em 2011 e 36\% foram consideradas insatisfatórias (Menck, Serafim, \& Oliveira, 2019). Efetivamente, em casos com alimentos contaminados por agrotóxicos, a vigilância em saúde deve realizar além do monitoramento, a interdição de alimentos suspeitos ou confirmados, a determinação de rotas de distribuição e de pontos de venda, medidas importantes para prevenção de agravos na saúde humana e ambiental. Após estas intervenções a identificação retrospectiva da cadeia produtiva que levou o uso do contaminante deve ser realizada para descobrir os fatores causais e propor medidas para evitá-los futuramente (Camara \& Corey, 1994).

Já o monitoramento sistemático de resíduos de agrotóxicos em mananciais e sistemas de abastecimento de água, é relevante pois a água pode ser contaminada por diferentes formas, pulverização, limpeza de equipamentos, chuva e descarte de embalagens. Contudo, metodologicamente, análises de metais pesados e alguns princípios ativos de agrotóxicos são caras e requerem tecnologias que nem todas as regiões possuem (Neto et al., 2014). Em 2008, entre todos os sistemas de abastecimento de água cadastrados no Sisagua, apenas $24 \%$ apresentavam informações sobre o controle da qualidade da água para os parâmetros de contaminação por agrotóxicos e somente $0,5 \%$ tinha dados sobre a vigilância da qualidade da água para tais substâncias, mesmo sendo uma responsabilidade da área da saúde (Fernandes Neto et al., 2010).

Já sobre a identificação da elaboração de Planos de Ação Estadual para Vigilância de Populações Expostas a Agrotóxicos (Freitas \& Garibotti, 2020; Martins et al., 2016), percebe-se o atendimento da recomendação do MS para elaboração de propostas de ações da VSPEA, ocorrida após a publicação da Portaria MS/GM nº 2.938, e do repasse do recurso financeiro aos Fundos Estaduais de Saúde e do Distrito Federal (Ministério da Saúde, 2018). A organização destes planos reflete o compromisso dos estados do Rio Grande do Sul e da Bahia com o desenvolvimento das ações de proteção e a promoção da saúde, prevenção de doenças e agravos, análise de situação e com o monitoramento da saúde das populações expostas ou potencialmente expostas.

Os trabalhos avaliados também apontaram a realização de atividades de educação em saúde para a população exposta 
como potencialidade para o fortalecimento das ações de vigilância em saúde. Considera-se que estas ações são axiomáticas pois os expostos precisam saber dos riscos a que estão submetidos, a fim de ser capazes de prevenir ou mitigar a exposição e até mesmo auxiliar em medidas de vigilância, prevenção e controle. Ações educativas comprometidas com a realidade local sensibilizam para elaboração de medidas em prol de uma produção e consumo menos prejudicial para os ecossistemas (Beserra et al., 2010). Ressalta-se que incumbe ao poder executivo desenvolver ações educativas de forma sistemática, visando atingir a população exposta a agrotóxicos divulgando o controle do uso, uso adequado e manejo alternativo de pragas e doenças a fim de reduzir os efeitos prejudiciais.

Outra medida de potencial identificada foram as parcerias intersetoriais (Martins et al., 2016; Mota et al., 2016; Santos et al., 2015), entre elas a atuação da Emater esteve em evidência. Este órgão atua em conjunto com a saúde e os trabalhadores rurais realizando atividades in loco direcionadas ao desenvolvimento rural (Santos et al., 2015). Outros estudos também apontam o benefício da intersetorialidade no enfrentamento da problemática da exposição e uso de agrotóxicos (Pignati et al., 2014; Riquinho \& Hennington, 2014).

A complexidade das externalidades das exposições a agrotóxicos exige articulação com outros setores a fim de garantir o direito a saúde e a integralidade de atenção. A intersetorialidade é, portanto, uma ferramenta indispensável para ações de curto, médio e longo prazo (Mota et al., 2016). Parcerias com outros setores agregam oportunidades para que a temática entre na agenda dos municípios, para gerar sinergia de saberes, competências, relações e experiências na defesa das populações expostas. Ainda a interação entre os diversos pontos de atenção à saúde, prioritariamente as vigilâncias e a Atenção Primária em Saúde, com os demais setores e com a sociedade civil, torna-se um espaço para construção de ambientes saudáveis, onde a promoção à saúde pode vir a se antecipar efetivamente aos agravos, superando o modelo de assistência curativista e fomentando a melhoria da qualidade de vida dos envolvidos.

O número reduzido de profissionais foi indicado como um obstáculo para a efetivação das ações de vigilância em saúde por três estudos (Neto et al., 2014; Rigotto et al., 2012; Santos et al., 2015). Ouve relatos de que em alguns municípios as ações de vigilância em saúde recaem sobre um único sujeito, de maneira que, quando este se desvincula do serviço, as atividades específicas desta área ficam descontinuadas (Santos et al., 2015). Soma-se a isso a grande demanda de trabalho, dificultando a implementação de ações mais amplas com foco na relação saúde e ambiente.

Outro ponto de fragilidade reconhecido por três artigos foi a limitação no acesso a dados sobre o uso de agrotóxicos (Faria et al., 2007; Neto et al., 2014; Santos et al., 2015). Informações sobre a quantidade de produtos utilizados e suas especificações, como a toxicologia e grupo químico são fundamentais para auxiliar na determinação das exposições, contudo foi observado dificuldade na obtenção desse tipo de informação. Dados sobre venda e consumo poderiam ser obtidas, exemplificadamente, nos Receituários Agronômicos e no site do Sindicato Nacional da Indústria de Produtos para Defesa Agrícola (SINDAG), porém essas bases não possuem base de dados completa abertas ao público.

O site do SINDAG informava a venda de agrotóxicos por unidade de federação, informando a quantidade de produto comercializada por ingrediente ativo e valor de vendas, porém a partir de 2006 essa informação não se encontra mais disponível por unidade de federação (Faria et al., 2007). Já o uso do Receituário Agronômico vem se distanciando do seu objetivo original, de controle do uso de agrotóxicos. De maneira geral, sua emissão apresenta irregularidades, muitas vezes ocorre sem visita a lavoura, levando ao uso de agrotóxicos inadequados ou não autorizados para algumas culturas, ou mesmo na ausência de pragas (Carneiro, et al., 2015). Há também carência de um sistema informatizado nacional que permita agregação e análise das informações geradas dos Receituários Agronômicos a fim de subsidiar elaborações de mapas do uso, identificação das pragas existentes e dos agrotóxicos mais utilizados nas lavouras, informações interessantes para o planejamento de ações de diversos setores (Carneiro, et al., 2015). A falta de sistematização destes dados dificulta a mensuração do impacto da exposição, além disso o lobby exercido pelas grandes corporações dificulta o acesso a informação (Dutra \& Ferreira, 2017). 
No que concerne a falta de interesse político para efetivação das ações de vigilância em saúde junto a cadeia produtiva do agronegócio (Neto et al., 2014; Santos et al., 2015), percebe-se o reconhecimento do setor como uma importante fonte geradora de empregos e renda, assim a literatura sugere que ações mais severas e sistematizadas podem impactar no interesse de grupos econômicos em continuar investindo na região, o que pode desmoralizar iniciativas institucionais (Neto et al., 2014). Esse contexto implica de modo direto na governança da saúde e ambiental, qual encontra dificuldades em traçar estratégias de desenvolvimento sustentável (Buss et al., 2012).

Trabalhar e projetar ações que vão de encontro com os argumentos do mercado ainda é um desafio que precisa ser assumido por todos, sociedade, produtores, vendedores de insumos e principalmente o Estado, que deve exercer a defesa dos direitos e da saúde da população. Ademais, observa-se o desafio de aliar estratégias estabelecidas pela macropolítica com as demandas emergentes dos territórios, priorizando o tema e outros problemas ligados ao espaço a agenda dos agrotóxicos (Santos et al., 2015).

Por fim, destacam-se como limitações desta pesquisa a carência de estudos em outras regiões do país, tendo em vista a heterogeneidade dos territórios brasileiros e de investigações com objetivos específicos voltados avaliações do processo de trabalho da vigilância em saúde para populações expostas a agrotóxicos.

\section{Conclusão}

Por meio desta revisão foi possível identificar que ações de vigilância em saúde para populações expostas a agrotóxicos, quando existentes, envolvem a notificação de intoxicações por agrotóxicos, suporte para diagnóstico e tratamento de intoxicações, uso de sistemas de informação para gerenciamento de dados, capacitação técnica de profissionais da saúde, monitoramento de contaminações de água e alimentos e elaboração de Planos de Ação Estadual para Vigilância de Populações Expostas a Agrotóxicos.

Pode-se observar que efetivar as práticas de vigilância em saúde para este público deve envolver processos intersetoriais e interdisciplinares que incorporem em análises situacionais locais o processo produtivo do agronegócio e a identificação de informações sociais, ambientais e de saúde.

Diante da importância da atuação da vigilância em saúde e da magnitude das exposições, espera-se com os resultados desta auxiliar gestores e profissionais no desenvolvimento de estratégias de prevenção e promoção da saúde da população expostas a agrotóxicos, bem como fomentar a importância de estudar ações de saúde orientadas a estes grupos.

Sugere-se a realização de novos estudos que identifiquem in loco ações e lacunas da vigilância em saúde de populações expostas a estes produtos, bem como estudos que divulguem estratégias pertinentes e resolutivas de proteção a saúde dos expostos.

\section{Referências}

Abreu, R. M., \& Tavares, F. G. (2016). Panorama do uso de agrotóxicos na Bahia: desafios para a vigilância à saúde, Revista Baiana de Saúde Pública, 40(2), 91-113. https://doi.org/10.22278/2318-2660.2016.v40.n0.a2696.

Albuquerque, P. C. C., Gurgel, I. G. D., Gurgel, A. M., Augusto, L. G. S., \& Siqueira, M. T. (2015). Sistemas de informação em saúde e as intoxicações por agrotóxicos em Pernambuco. Revista Brasileira de Epidemiologia, 18(3), 666-678. https://doi.org/10.1590/1980-5497201500030012.

Barros, J. R. M., \& Barros, A. L. M. (2005). A geração de conhecimento e o sucesso do agronegócio brasileiro. Revista de Política Agrícola, 14(4). https://seer.sede.embrapa.br/index.php/RPA/article/view/546/pdf.

Beserra, E. P., Alves, M. D. S., Pinheiro, P. N. C., \& Vieira, N. F. C. (2010). Educação ambiental e enfermagem: uma integração necessária. Revista Brasileira Enfermagem, 63(5), 848-852. https://doi.org/10.1590/S0034-71672010000500026.

Buss, P. M., Machado, J. M. H., Gallo, E., Magalhães, D. P., Setti, A. F. F., Franco, N. F. A., \& Buss, D. F. (2012). Governança em saúde e ambiente para o desenvolvimento sustentável. Ciência \& Saúde Coletiva, 17(6), 1479-1491. https://doi.org/10.1590/S1413-81232012000600012. 
Camara, V. M., \& Corey, G. (1994). Epidemiologic Surveillance for Substances Banned from Use in Agriculture. Bulletin of PAHO, 28(4). https://iris.paho.org/bitstream/handle/10665.2/26915/ev28n4p355.pdf?sequence=1\&isAllowed=y.

Carneiro, F. F., Augusto, L. G. S., Rigotto, R. M., Friedrich K., \& Búrigo, A. C. (Orgs.). (2015). Dossiê ABRASCO: Um alerta sobre os impactos dos agrotóxicos na saúde. Rio de Janeiro: EPSJV, São Paulo: Expressão Popular.

CEPEA - Centro de Estudos Avançados em Economia Aplicada. (2020). PIB do agronegócio brasileiro. https://www.cepea.esalq.usp.br/br/pib-do-agronegociobrasileiro.aspx.

Dutra, L. S., \& Ferreira, A. P. (2017). Associação entre malformações congênitas e a utilização de agrotóxicos em monoculturas no Paraná, Brasil. Saúde em Debate, 41(especial), 241-253. https://dx.doi.org/10.1590/0103-11042017s220.

EMBRAPA - Empresa Brasileira de Pesquisa Agropecuária. (2018). Trajetória da agricultura brasileira. https://www.embrapa.br/visao/trajetoria-da-agriculturabrasileira.

Faria, N. M. X., Facchini, L. A., Fassa, A. G., \& Tomasi, E. (2004). Trabalho rural e intoxicações por agrotóxicos. Cadernos de Saúde Pública, 20(5), 12981308. https://doi.org/10.1590/S0102-311X2004000500024.

Faria, N. M. X., Fassa, A. G., \& Facchini, L. A. (2007). Intoxicação por agrotóxicos no Brasil: os sistemas oficiais de informação e desafios para realização de estudos epidemiológicos. Ciência \& Saúde Coletiva, 12(1), 25-38. https://doi.org/10.1590/S1413-81232007000100008.

Fernandes Neto, M. L. (2010). Norma Brasileira de Potabilidade de Água: Análise dos parâmetros agrotóxicos numa abordagem de avaliação de risco. Tese de Doutorado, Fundação Oswaldo Cruz, Escola Nacional de Saúde Pública Sergio Arouca, Rio de Janeiro, RJ, Brasil. https://www.arca.fiocruz.br/handle/icict/2581.

Freitas, A. B., \& Garibotti, V. (2020). Caracterização das notificações de intoxicações exógenas por agrotóxicos no Rio Grande do Sul, $2011-2018$. Epidemiologia e Serviços de Saúde, 29(5), e2020061. https://dx.doi.org/10.1590/s1679-49742020000500009.

Garcia, E. G., \& Alves Filho, J. P. (2005). Aspectos de prevenção e controle de acidentes no trabalho com agrotóxicos. São Paulo: Fundacentro.

Hendges, C., Schiller, A. P., Manfrin, J., Macedo, Jr. E. K., Gonçalves Jr., A. C., \& Stangarlin, J. R. (2019). Human intoxication by agrochemicals in the region of South Brazil between 1999 and 2014, Journal of Environmental Science and Health, Part B. https://doi.org/10.1080/03601234.2018.1550300.

IBGE - Instituto Brasileiro de Geografia e Estatística. (2019). Censo agropecuário 2017: resultados definitivos. IBGE: Rio de Janeiro, 8, 1-105. https://biblioteca.ibge.gov.br/visualizacao/periodicos/3096/agro_2017_resultados_definitivos.pdf.

Jorge, M. H. P. M., Laurenti, R., \& Gotlieb, S. L. D. (2010). Avaliação dos sistemas de informação em saúde no Brasil. Cadernos Saúde Coletiva, 18(1), 7-18. http://www.cadernos.iesc.ufrj.br/cadernos/images/csc/2010_1/artigos/Modelo\%20Livro\%20UFRJ\%201-a.pdf.

Kreter, A. C., \& Souza Júnior, J. R. C. (2018). Carta de Conjuntura, n. 40, $3^{\circ}$ trimestre 2018: Economia Agrícola. Brasília-DF: IPEA. https://www.ipea.gov.br/cartadeconjuntura/index.php/2018/08/economia-agricola-4/.

Lacaz, F. A. C., Machado, J. M. H., \& Porto, M. F. S. (2002). Estudo da situação e tendências da vigilância em saúde do trabalhador no Brasil. Apoio financeiro: Organização Pan - Americana da Saúde (OPAS). Apoio logístico: ABRASCO. http://renastonline.ensp.fiocruz.br/sites/default/files/arquivos/recursos/E studo\%20da\%20Situa\%C3\%A7\%C3\%A3o\%20e\%20Tend\%C3\%AAncias\%20da\%20Vigil\%C3\%A2ncia\%20em\%20Sa\%C3\%BAde\%20do\%20Trabalhador\% 20no\%20Brasil.pdf.

Lei n ${ }^{\circ}$ 8.080, de 19 de setembro de 1990. (1990). Dispõe sobre as condições para a promoção, proteção e recuperação da saúde, a organização e o funcionamento dos serviços correspondentes e dá outras providências. Diário Oficial da União, Brasília, DF, 182, 20 set. Seção 1, p. 18055.

Martins, E. H. C. et al. (2016). Vigilância e atenção à saúde de populações expostas a agrotóxicos: metodologias e práticas integradas. Revista Baiana de Saúde Pública, 40(2), 166-174. http://rbsp.sesab.ba.gov.br/index.php/rbsp/article/view/2700/1902.

Marziale, M. H. P. (2015). Instrumento para recolección de datos revisión integrativa.

RedENSO (Red Internacional de Enfermería en Salud Ocupacional). http://gruposdepesquisa.eerp.usp.br/sites/redenso/wpcontent/uploads/sites/9/2019/09/Instrumiento_revision_litetarura_RedENSO_2015.pdf.

Menck, V. F., Serafim, M. P., \& Oliveira, J. M. (2019). Intoxicação do(a) trabalhador(a) rural por agrotóxicos: (sub)notificação e (in)visibilidade nas políticas públicas de 2001 a 2015. Segurança Alimentar e Nutricional. 26, e019001. https://doi.org/10.20396/san.v26i0.8651513.

Ministério da Saúde. Secretaria de Vigilância em saúde. Departamento de Saúde Ambiental e Saúde do Trabalhador. (2012). Documento Orientador para a Implementação da Vigilância em saúde de Populações Expostas a Agrotóxicos. Brasília: Ministério da Saúde.

Ministério da Saúde. Secretaria de Vigilância em saúde. Departamento de Vigilância em saúde Ambiental e Saúde do Trabalhador. (2016). Diretrizes nacionais para a vigilância em saúde de populações expostas a agrotóxicos. Brasília: Ministério da Saúde.

Ministério da Saúde. Secretaria de Vigilância em saúde. Departamento de Vigilância em saúde Ambiental e Saúde do Trabalhador. (2018). Agrotóxicos na ótica do Sistema Único de Saúde. Relatório Nacional de Vigilância em saúde de Populações Expostas a Agrotóxicos. Brasília: Ministério da Saúde. https://bvsms.saude.gov.br/bvs/publicacoes/relatorio_nacional_vigilancia_populacoes_expostas_agrotoxicos.pdf.

Mota, M. C. T., Cancio, J. A., Nobre, L. C. C., Coelho, A. J. F., Junior, C. A., Porto, C. L., Santos, G., Santos, J. F., \& Abreu, R. M. (2016). Experiência de ação integrada em emergência fitossanitária na região oeste da Bahia. Revista Baiana de Saúde Pública, 40(2), 188-198. https://doi.org/10.22278/23182660.2016.v40.n0.a2702.

Neto, E. N., Lacaz, F. A. C., \& Pignati, W. A. (2014). Vigilância em saúde e agronegócio: os impactos dos agrotóxicos na saúde e no ambiente. Perigo à vista!. Ciência \& Saúde Coletiva, 19(12), 4709-4718. https://dx.doi.org/10.1590/1413-812320141912.03172013. 
Neves, P. D. M., Mendonça, M. R., Bellini, M., \& Pôssas, I. B. (2020). Intoxicação por agrotóxicos agrícolas no estado de Goiás, Brasil, de 2005-2015: análise dos registros nos sistemas oficiais de informação. Ciência \& Saúde Coletiva, 25(7), 2743-2754. https://doi.org/10.1590/1413-81232020257.09562018.

Oliveira, M. L. F., Silva, A. A., Ballani, T. S. L, \& Bellasalma, A. C. M. (2003). Sistema de Notificação de Intoxicações: desafios e dilemas. In: Peres F., Moreira J. C., (Orgs.). É veneno ou é remédio? Rio de Janeiro: Fiocruz, 303-315.

Pereira A. S., Shitsuka, D. M, Shitsuka, F. J. P. R. (2018). Metodologia da pesquisa científica. UAB/NTE/UFSM: https://repositorio.ufsm.br/bitstream/handle/1/15824/Lic_Computacao_Metodologia-Pesquisa-Cientifica.pdf?sequence=1.

Pignati, W., Oliveira, N. P., \& Silva, A. M. C. (2014). Vigilância aos agrotóxicos: quantificação do uso e previsão de impactos na saúde-trabalho-ambiente para os municípios brasileiros. Ciência \& Saúde Coletiva, 19(12), 4669-4678. https://doi.org/10.1590/1413-812320141912.12762014.

Pires, D. X., Caldas, E. D., \& Recena, M. C P. (2005). Intoxicações provocadas por agrotóxicos de uso agrícola na microrregião de Dourados, Mato Grosso do Sul, Brasil, no período de 1992 a 2002. Cadernos de Saúde Pública, 21(3), 804-814. https://www.toxicologia.unb.br/img_banners_publicidade/350.pdf.

Resolução No 588, de 12 de julho de 2018. (2018). Fica instituída a Política Nacional de Vigilância em saúde (PNVS), aprovada por meio desta resolução. Diário Oficial da União, Brasília, DF, 155, 13 ago. Seção 1, p. 87-90.

Rigotto, R. M., Carneiro, F. F., Marinho, A. M. C. P., Rocha, M. M., Ferreira, M. J. M., Pessoa, V. M., Teixeira, A. C. A., Silva, M. L. V., Braga, L. Q. V., \& Teixeira, M. M. (2012). O verde da economia no campo: desafios à pesquisa e às políticas públicas para a promoção da saúde no avanço da modernização agrícola. Ciência \& Saúde Coletiva, 17(6), 1533-1542. http://dx.doi.org/10.1590/S1413-81232012000600017.

Riquinho, D. L., \& Hennington, E. A. (2014). Aderir ou resistir ao cultivo do tabaco? histórias de trabalhadores rurais de localidade produtora no sul do Brasil. Ciência \& Saúde Coletiva, 19(10), 3981-3990. http://doi.org/10.1590/1413-812320141910.08792014.

Santos, V. C. F., Ruiz, E. N. F., Riquinho, D. L., \& Mesquita, M. O. (2015). Saúde e ambiente nas políticas públicas em municípios que cultivam tabaco no sul do Brasil. Revista Gaúcha de Enfermagem, 36(especial), 215-223. https://www.scielo.br/pdf/rgenf/v36nspe/0102-6933-rgenf-36-spe-0215.pdf.

Silva, D. B. (2012). Sustentabilidade no Agronegócio: dimensões econômica, social e ambiental. Revista Comunicação \& Mercado/UNIGRAN, 1(3), 23-34.

Silvério, A. C. P., Martins, I., Nogueira, D. A., Mello, M. A. S., Loyola, E. A. C., \& Graciano, M. M. C. (2020). Avaliação da atenção primária à saúde de trabalhadores rurais expostos a praguicidas. Revista de Saúde Pública, 54, 09. https://doi.org/10.11606/s1518-8787.2020054001455.

Taveira, B. L. S., \& Albuquerque, G. S. C. (2018). Análise das notificações de intoxicações agudas, por agrotóxicos, em 38 municípios do estado do Paraná. Saúde Debate, 42(especial 4), 211-222. http://doi.org/10.1590/0103-11042018S417.

The Joanna Briggs Institute. (2015). The Joanna Briggs Institute Reviewers’ Manual 2015: Methodology for JBI Scoping Reviews. South Australia: The Joanna Briggs Institute.

WorldAtlas. (2017). Top pesticide using countries. https://www.worldatlas.com/articles/top-pesticide-consuming-countries-of-the-world.html. 